\title{
fMRI assessment of small animals' phobia using virtual reality as stimulus
}

\author{
Miriam Clemente, Beatriz Rey, Mariano Alcañiz \\ I3BH/LabHuman, Universitat Politècnica de València \\ Valencia, Spain \\ mclemente@labhuman.i3bh.es
}

\author{
Juani Bretón-López, Cristina Botella \\ I3BH/Lapsitec, Universitat Jaume I \\ Castellón de la Plana, Spain
}

\author{
Aina Rodríguez-Pujadas, Alfonso Barros-Loscertales, \\ César Ávila \\ Departamento de Psicología Básica, Clínica y Psicobiología, \\ Universitat Jaume I \\ Castellón de la Plana, Spain
}

\author{
Rosa M. Baños \\ I3BH/Lapsitec, Universitat de València \\ Valencia, Spain
}

anxiety, which refers to the fear you feel when you are expecting to find the animal object of your phobia. Regarding other image techniques, [6] used EEG to investigate spider phobia, using pictures as stimulus.

In the field of phobias, Virtual Reality (VR) has been repeatedly used to treat the disorder, but up to our knowledge, it has not been used for the assessment of the disturbance yet. VR is a technology that comprises computer-generated simulations of reality [7]. One of the main advantages of VR is that it allows the patient to interact with the phobic object or situation, as if they were real and he were there with the feared animals.

The VR exposure therapy (VRET) has been widely used in the treatment of specific phobias [8], such as acrophobia, claustrophobia, spider phobia, fear of driving and fear of flying. However, until now VR has not yet been used inside the fMRI as a stimulus to assess the responses of phobic subjects in the presence of the feared elements.

We propose that the use of VR as stimulus in fMRI environments will entail the same advantages to the phobia evaluation that it brought to the phobia treatment. In order to validate this proposal, our target in the present work is to examine if VR can be used for the assessment of the phobia, provoking a more realistic and immersive situation than the view of a still photograph. We have used virtual environments where the subject can navigate freely. Our main hypothesis is that the brain areas activated with these environments will be coherent with results from previous studies based on pictures or videos of real animals. This could be the base of a future evaluation system (similar to the existing treatment protocols) to assist the therapist in the assessment of the disorder.

\section{MATERIALS AND METHODS}

[2] used film excerpts of real spiders as stimulus and excerpts of real butterflies as control. Reference [3] studied the effect of a cognitive therapy using pictures of spiders, comparing them with images that provoked fear, disgust and neutral ones. Reference [4] also used pictures as stimulus in an fMRI study over phobic and non-phobic subjects to evaluate the activation of the amygdala. Reference [5] analyzed the anticipatory

\section{A. Subjects}

For this study, we recruited 11 right-handed phobic women, aged between 20 and 35 (mean age 24.64). None of them had any other medical or psychological disorders, apart from the phobia. The diagnosis and assessment phase was carried out by 
expert clinicians who were also the therapists for the participants. Ethical approval was obtained from the authors' institution, and each subject signed a written informed consent prior to participation.

\section{B. Environments}

The virtual environments were programmed using GameStudio software. We have divided the task into three experimental conditions, all of them involving a room where the subject can navigate freely. In the first of these conditions ('CLEAN'), the patient navigates through a common clean bedroom. In the second condition ('DIRTY'), the navigation is performed through the same room, but this time dirty and darker, giving the subject the feeling that the feared animal could appear in any moment. In the last condition ('PHOBIC'), the subject navigates through the same dirty room, but this time there appeared spiders and cockroaches. Each of the three experimental conditions was repeated six times in a counterbalanced order to prevent effects produced by the order in which they were presented.

\section{C. fMRI Procedures}

All the subjects were scanned in a 1.5 Tesla Siemens Avanto Magnetic Resonance scanning device. To display the environments, we used MRI-compatible video goggles and for the navigation an adapted joystick. First, sagittal T1-weighted structural images were acquired (224 x 256 matrix covering the brain with 176 contiguous $1 \mathrm{~mm}$ slices, repetition time of TR = $11 \mathrm{~ms}$, echo time of $\mathrm{TE}=4.94 \mathrm{~ms}$, flip angle of $\mathrm{FA}=15^{\circ}$, voxel size $=1.04 \times 1.04 \mathrm{~mm}$ ). Then, the functional scanning was launched, synchronized with the virtual environments. Functional images were obtained using a T2* single-shot echoplanar imaging (EPI) sequence. We used 30 contiguous $4.2 \mathrm{~mm}$ interleaved axial slices (parallel to the AC-PC line, that traverses the brain from the anterior to the posterior commissure) covering the entire volume of the brain with a 64 x 64 matrix $\left(\mathrm{TR}=2000 \mathrm{~ms}, \mathrm{TE}=30 \mathrm{~ms}, \mathrm{FA}=90^{\circ}\right.$, voxel size $=3.5 \times 3.5 \mathrm{~mm})$.

\section{Data Analysis}

We have used the Statistical Parametric Mapping software (SPM8) for the analysis of the fMRI data, launched with the 7.1 version of Matlab. After the preprocessing of the data, a first fixed-effect level analysis was carried out. Then, we performed group tests at a second level random effect analysis. We tested for task related activation by performing a onesample t-test including contrast images of estimated parameters for the differences of interest between conditions. We obtained three contrasts: the results that show the brain activations for the phobic stimulus are contained in the "phobic $>$ clean" contrast, the "phobic $>$ dirty" contrast shows phobic activations avoiding the anxiety feeling caused by the dirtiness of the room, and the "dirty>clean" contrast contains the anxiety related activations. All contrasts at group level were considered if more than 10 adjacent voxels passed the statistical threshold of $p<0.005$ (uncorrected). These results were corrected at $\mathrm{p}<0.05$ using AlphaSim correction (combined height threshold $\mathrm{p}<0.005$ and a minimum cluster size $=25)$ [9].

\section{RESULTS}

We selected the contrast "phobic $>$ clean" and looked for the main activated brain regions. We found activations in the left occipital inferior lobe and middle occipital gyrus bilaterally among others (see superior part of Table 1 and Fig. 1). Other brain regions which displayed significant activations during the task were the cuneus bilaterally, the superior frontal gyrus and the precuneus. In the middle and lower parts of Table 1, we can observe the rest of the results obtained for the "phobic $>$ dirty" (inferior occipital lobe bilaterally, and the left superior and middle frontal lobe) and "dirty > clean" (left superior occipital lobe, and right middle frontal gyrus, middle occipital gyrus and cingulate) contrasts. Apart from the anatomical area and hemisphere, in the table the values for the location and $\mathrm{T}$ score of the maximum for each area, the size of the cluster activated in each area and the $\mathrm{p}$ value used as threshold are shown.

\section{DISCUSSION}

The main goal of our study was to analyze the brain areas activated due to phobic stimulus during navigation through a virtual environment in three different experimental conditions (CLEAN, DIRTY and PHOBIC). One of the main results for the purposes of the study are those obtained when comparing brain activations between phobic and clean conditions ("phobic>clean"), which are those that reflect the fear and anxiety felt by the subjects due to the phobic stimulus when compared with a emotionally neutral situation. Both the phobic and dirty situations may generate anxiety in the participant. However, in the dirty condition the anxiety is generated by the fact of being in a threatening room (because of the dirtiness of the room, the participant may feel that is a dangerous place to be in) and in the phobic condition there are phobic stimuli, spiders and cockroaches, that will generate a phobic specific activation in the brain. In the "phobic >clean" comparison the activations may be caused by both factors. The activations obtained in the "phobic >dirty" contrast would be directly related to the phobia itself, and not to the anxiety feeling.

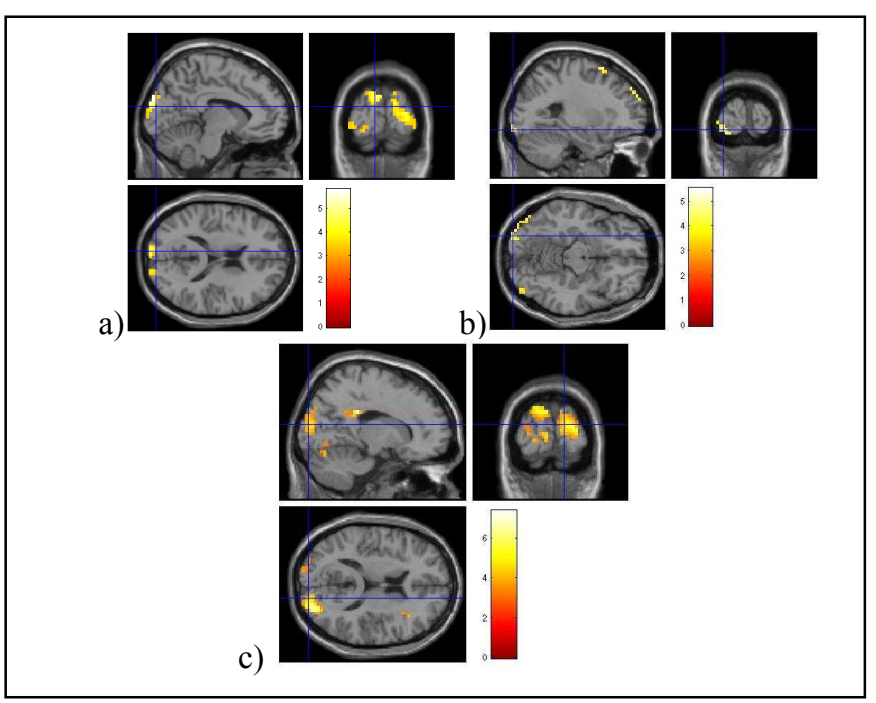

Figure 1. Brain activations for the (a)"phobic >clean", (b) "phobic $>$ dirty" and (c) "dirty>clean" contrasts. 
TABLE I. BRAIN AREA ACTIVATION RESULTS

\begin{tabular}{|c|c|c|c|c|c|c|}
\hline \multirow[b]{2}{*}{ Contrast } & \multicolumn{6}{|c|}{ Brain Area Activation Results } \\
\hline & Anatomical Region & Hemisphere & $(x, y, z)$ & T score & $\begin{array}{c}\text { Cluster } \\
\text { Size }\end{array}$ & $p$ \\
\hline \multirow{8}{*}{$\begin{array}{l}\text { "Phobic }>\text { Clean" } \\
\text { Contrast }\end{array}$} & Occipital Inferior Lobe & $\mathrm{L}$ & $(-22,-98,-12)$ & 4,19 & 36 & $\mathrm{p}<0.05$ corrected \\
\hline & Middle Occipital Gyrus (BA19) & $\mathrm{L}$ & $(-54,-77,-4)$ & 5,21 & 29 & $\mathrm{p}<0.05$ corrected \\
\hline & Middle Occipital Gyrus & $\mathrm{R}$ & $(31,-77,0)$ & 4,76 & 175 & $\mathrm{p}<0.05$ corrected \\
\hline & Cuneus & $\mathrm{R}$ & $(20,-91,9)$ & 4,01 & 36 & $\mathrm{p}<0.05$ corrected \\
\hline & BA18 & $\mathrm{R}$ & $(26,-96,6)$ & $-11,64$ & 28 & $\mathrm{p}<0.05$ corrected \\
\hline & Cuneus & $\mathrm{L}$ & $(-8,-95,30)$ & 5,82 & 55 & $\mathrm{p}<0.05$ corrected \\
\hline & Superior Frontal Gyrus & $\mathrm{R}$ & $(20,49,42)$ & 4,52 & 13 & $\mathrm{p}<0.005$ uncorrected \\
\hline & Precuneus & $\mathrm{L}$ & $(-1,-46,68)$ & 4,59 & 31 & $\mathrm{p}<0.05$ corrected \\
\hline \multirow{4}{*}{$\begin{array}{l}\text { "Phobic > } \\
\text { Dirty" contrast }\end{array}$} & Inferior Occipital Lobe & $\mathrm{L}$ & $(-26,-98,-12)$ & 5,52 & 54 & $\mathrm{p}<0.05$ corrected \\
\hline & Inferior Occipital Lobe & $\mathrm{R}$ & $(48,-84,-8)$ & 4,43 & 22 & $\mathrm{p}<0.005$ uncorrected \\
\hline & Superior Frontal Lobe & $\mathrm{L}$ & $(-22,56,34)$ & 4,51 & 18 & $\mathrm{p}<0.005$ uncorrected \\
\hline & Middle Frontal Lobe & $\mathrm{L}$ & $(-26,14,63)$ & 5,25 & 18 & $\mathrm{p}<0.005$ uncorrected \\
\hline \multirow{4}{*}{$\begin{array}{l}\text { "Dirty > Clean" } \\
\text { contrast }\end{array}$} & Superior Occipital Lobe & $\mathrm{L}$ & $(-15,-91,30)$ & 5,69 & 201 & $\mathrm{p}<0.05$ corrected \\
\hline & Middle Frontal Gyrus & $\mathrm{R}$ & $(24,53,-8)$ & 5,23 & 39 & $\mathrm{p}<0.05$ corrected \\
\hline & Middle Occipital Gyrus & $\mathrm{R}$ & $(27,-84,13)$ & 6,81 & 184 & $\mathrm{p}<0.05$ corrected \\
\hline & Cingulate Gyrus & $\mathrm{R}$ & $(17,-35,30)$ & 7,40 & 14 & $\mathrm{p}<0.005$ uncorrected \\
\hline
\end{tabular}

One of the most important activated areas in the "phobic >clean" contrast is the occipital lobe, more specifically, activated in its left inferior area and in the middle lobe bilaterally. The occipital lobe mainly controls the visual areas, which are necessary for the performance of a navigation task. In a similar study, it was found a similar activation in this area when subtracting spiders' minus butterflies' contrasts [2]. They concluded that this activation was related with enhanced visual attention to the phobic stimuli, and support vigilance functions in anxiety [10].

More recently, there have been several fMRI [3, 5] and PET [6] studies among phobic and non-phobic subjects that have also found activation in the visual cortex. In fact, [5] justified it as likely to be caused by the attention subjects put on the visual input that reflect an "increase in the processing of the cue but also the expectation of behaviorally relevant sensory input".

The other important activation is found in the superior frontal gyrus, related to the feeling of self-awareness [11], which is increased when the phobic subject watches the animal that provokes his fear. During a resting situation, the subject relaxes and is less conscious of himself. But when the phobic person finds himself in a fearful situation, his alert state increases, trying to inhibit his reaction in front of the phobic stimulus [2]. The natural reply to this stimulus is to avoid the fear response it provokes over him, and to do so he controls his mind and body, increasing the consciousness he has of himself. Reference [2] discussed the relation of the frontal activations with the voluntary self-regulation of emotion. They pointed out that the phobic subjects activated their prefrontal areas when attempting to control their fear before the film excerpts of spiders. Another explanation is given by [11], who analyzed the subjective awareness feeling and its relation with the frontal areas of the brain. They remarked how when watching an absorbing movie or being involved in a highly demanding sensory task (as is in our case the virtual navigation through an immersive environment) the strong subjective feeling is of "losing the self", or, as they explained, of disengaging from self-related reflective processes. Accepting this state, the increase in the self-awareness feeling during the visualization of phobic stimulus in a highly demanding navigation task is clearly related to the higher feeling of yourself when "fighting" the fear. In words of [6], increased activation in the superior frontal cortex might reflect patients' urge to flee during the confrontation with the feared object; and this link between the sensorimotor system and the affective/cognitive function is in line with the theory about embodied cognition [12]. In conclusion, we can consider this activation essentially related to the phobia.

We also found activity in the cuneus and the precuneus. The cuneus is related to visual processing, which is directly associated with the sense of presence that the subject feels while navigating through a virtual environment [13]. On the other side, the precuneus is related to self-consciousness, such as reflective self-awareness, that involves rating your own personality traits [14].

Although one of the areas most commonly related to phobias is the amygdala, it is not activated in our results. 
Several previous studies have been conducted to find the pattern of activation of this area [2, 4], concluding that it suffers habituation over time [4]. Reference [5] discussed that the amygdala activation may occur during brief presentations of the phobogenic stimuli and in the induction of rapid behavioral responses more than in the sustained and explicit processing of the threatening stimuli. In our case, the use of periods of navigation (block design) instead of pictures may be the cause of not detecting activation in this area.

Regarding the results for the "phobic $>$ dirty" comparison, we found that the inferior occipital lobe played a major role in the fear response to the phobic stimulus, bilaterally. This is in concordance with the results obtained for the "phobic $>$ clean" contrast, where we pointed out the relation of this area with the phobic response. As we have already said, the occipital lobe is related to enhance visual attention to the phobic stimuli [10]. The other important activation is located in the superior and middle frontal lobe, result also contained in our previous contrast, due to its relation with the feeling of self-awareness and the action of the sensory system [11]. As we can see, the main results that we pointed out related to the phobia are still activated when we restrict the conditions of the contrast to avoid the anxiety results.

Regarding the "dirty>clean" contrast, the self-awareness is still high, due to the greater fear of finding a spider or cockroach when navigating through a dark and dirty environment than when navigating through a clean one, which results in the activation of the middle frontal gyrus. The activation of the occipital lobe is maintained here due to the higher visual processing when expecting the appearance of a feared animal. The last activation was located in the cingulate gyrus, which [2] pointed out to be mainly associated with the cognitive/internal generation of emotional state by evoking visual imagery or memories. As aforementioned, the activations in this contrast are due to the evocation of fear, not to the exposition to it; so the meaning of the activation in the cingulate gyrus is clear as a generator of emotional evocations.

In conclusion, we have obtained with VR similar results in terms of fMRI brain activations to those obtained using real stimuli. In fact, the main activations we found in the occipital and frontal areas are coherent with those found in previous studies conducted with spider phobic subjects using pictures or videos of real animals as stimuli. Moreover, the activation in the cuneus could be related to the sense of presence elicited in the subjects because of the navigation through the virtual environment. This finding opens the door to deeper investigations over the phobias, due to the fact that VR allows recreation of normal life scenes in a more realistic and interactive way, that are impossible to achieve with other techniques. This kind of situations could allow, for example, the study over subjects with a mild phobia, whose fear cannot be excited only by the use of photographs.

\section{ACKNOWLEDGMENT}

This study was funded by the Ministerio de Educación y Ciencia Spain, Project Game Teen (TIN2010-20187) and partially by projects Consolider-C (SEJ2006-14301/PSIC), "CIBER of Physiopathology of Obesity and Nutrition, an initiative of ISCIII", the Excellence Research Program PROMETEO (Generalitat Valenciana. Conselleria de Educación, 2008-157) and the Consolider INGENIO program (CSD2007-00012). The work of M.C. was supported by the Generalitat Valenciana under a VALi $+d$ Grant.

\section{REFERENCES}

[1] American Psychiatric Association, "Diagnostic and Statistical Manual of Mental Disorders," 4th ed., text revision. Washington, DC: American Psychiatric Association, 2000.

[2] V. Paquette, J. Lévesque, B. Mensour, J.M. Leroux, G. Beaudoin, P. Bourgouin, and M. Beauregard, "Change the mind and you change the brain: effects of cognitive-behavioral therapy on the neural correlates of spider phobia," Neuroimage, vol. 18, pp. 401-409, 2003.

[3] A. Schienle, A. Schäfer, A. Hermann, S. Rohrmann, and D. Vailt, "Symptom provocation and reduction in patients suffering from spider phobia," Eur Arch Psychiatry Clin Neurosci, vol. 257, pp. 486-493, 2007.

[4] C.L. Larson, H.S. Schaefer, G.J. Siegle, C.A.B. Jackson, M.J. Anderle, and R.J. Davidson, "Fear Is Fast in Phobic Individuals: Amygdala Activation in Response to Fear-Relevant Stimuli," Biol Psychiatry, vol. 60, pp. 410-417, 2006.

[5] T. Straube, H.J. Mentzel, and W.H.R. Miltner, "Waiting for spiders: brain activation during anticipatory anxiety in spider phobics," Neuroimage, vol. 37, pp. 1427-1436, 2007.

[6] W. Scharmüller, V. Leutgeb, A. Schäfer, A. Köchel, and A. Schienle, "Source localization of late electrocortical positivity during symptom provocation in spider phobia: An sLORETA study," Brain Res, vol. 1397, pp. 10-18, 2011.

[7] R.M. Baños, V. Guillen, S. Quero, A. García-Palacios, M. Alcañiz, and C. Botella, "A virtual reality system for the treatment of stressrelated disorders: A preliminary analysis of efficacy compared to a standard cognitive behavioral program," Int J Human-Computer Studies, vol. 69, pp. 602-613, 2011

[8] M. Krijn, P.M.G. Emmelkamp, R.P. Olafsson, and R. Biemond, "Virtual reality exposure therapy of anxiety disorders: A review," Clinical Psychology Review, vol. 24, pp. 259-281, 2004

[9] X.W. Song, Z.Y. Dong, X.Y. Long, S.F. Li, X.N. Zuo, C.Z. Zhu, Y. He, C.G. Yan, and Y.F. Zang, "REST: A Toolkit for Resting-State Functional Magnetic Resonance Imaging Data Processing," PLoS ONE, vol. 6, 2011.

[10] M. Fredrikson, G. Wik, P. Annas, K. Ericson, and S. Stone-Elander, "Functional neuroanatomy of visually elicited simple phobic fear: additional data and theoretical analysis," Psychophysiology, vol. 32, pp. 43-48, 1995.

[11] I. Goldberg, M. Harel, and R. Malach, "When the brain loses its self: prefrontal inactivation during sensorimotor processing," Neuron, vol. 50, pp. 329-339, 2006

[12] F. Garbarini, and M. Adenzato, "At the root of embodied cognition: cognitive science meets neurophysiology," Brain Cogn., vol. 56, pp. 100-106, 2004.

[13] D. Perani, F. Fazio, N.A. Borghese, M. Tettamanti, S. Ferrari, J. Decety, and M.C. Gilardi, "Different brain correlates for watching real and virtual hand actions," NeuroImage, vol. 14, pp. 749-758, 2001.

[14] H.C. Lou, B. Luber, M. Crupain, J.P. Keenan, M. Nowak, T.W. Kjaer, H.A. Sackeim, and S.H. Lisanby, "Parietal cortex and representation of the mental Self," Proceedings of the National Academy of Sciences of the United States of America, vol. 101, pp. 6827-6832, 2004. 